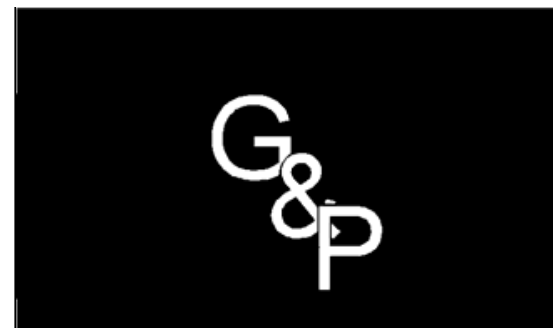

\title{
GERENCIAMENTO PELAS DIRETRIZES E O GERENCIAMENTO POR OBJETIVOS: UMAANÁLISE COMPARATIVA
}

\section{GESTÃO} \&

\section{PRODUÇÃO}

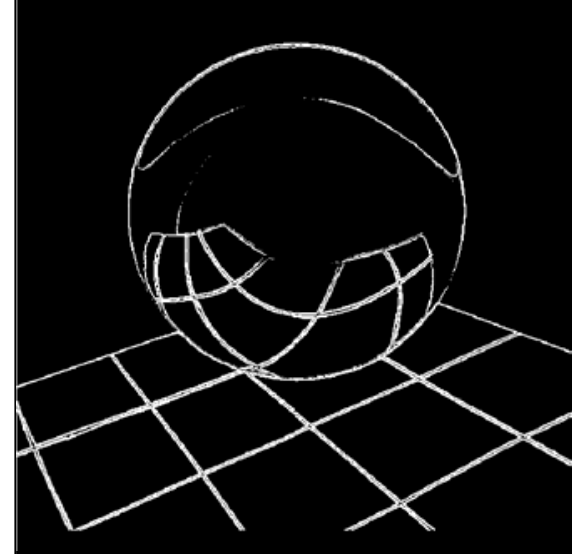

v.2, n.3, p. 331-338, dez.1995
João Batista Turrioni

Doutorando do Departamento de Engenharia de Produção

Escola Politécnica - USP

Professor Assistente do Departamento de Engenharia de Produção da Escola Federal de Engenharia de Itajubá - MG

Pedro Luiz de Oliveira Costa Neto

Departamento de Engenharia de Produção Escola Politécnica - USP

\section{Resumo}

A realidade brasileira e a velocidade do processo de difusão do modelo de gerenciamento da Qualidade Total e suas respectivas técnicas têm gerado algumas complexidades. Entre elas está a grande dificuldade da alta administração em conseguir que a Gerência da Qualidade Total realmente atinja os objetivos pretendidos com sua implementação. Este trabalho visa abrir a discussão sobre um método para prevenir essa dificuldade, o Gerenciamento pelas Diretrizes, analisando este modelo por meio de uma comparação com o mais tradicional, e em geral mais conhecido, Gerenciamento por Objetivos.

\section{Palavras-chave: Gerência da Qualidade Total, Gerenciamento pelas Diretrizes, Gerenciamento por Objetivos.}

\section{Introdução}

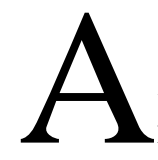

realidade mundial atual é caracterizada por avanços tecnológicos e mudanças sociais muito rápidas, exigindo das organizações flexibilidade a essas mudanças e respostas imediatas.

No Brasil, a realidade não é diferente e muitos esforços estão sendo desenvolvidos na direção da preparação de nossas organi- zações, dentre os quais podemos destacar o Programa Brasileiro da Qualidade e Produtividade (PBQP) que, por meio de um de seus projetos básicos, o Projeto de Especialização em Gestão da Qualidade (PEGQ), vem disseminando e implantando em organizações brasileiras métodos modernos de Gestão da Qualidade.

Por esta via, questões como o comprome- 
timento com a qualidade, o envolvimento da alta administração, a redução de níveis hierárquicos e o rompimento de barreiras administrativas, além de técnicas estatísticas e gerenciais para a qualidade, como o Desdobramento da Função Qualidade (QFD), Controle Estatístico de Processo (CEP), Análise dos Modos e Efeitos de Falha (FMEA), Sistemas de Garantia da Qualidade (ISO 9000), entre várias outras, têm sido largamente difundidas.

Entretanto, não se tem dado a devida ênfase à maneira pela qual determinações da alta administração da empresa possam ser eficaz e eficientemente executadas pelos demais níveis da organização, o que leva, com freqüência, a distorções ou incompreensões quanto ao que, de fato, se deseja realizar. Numa tentativa de contribuir para o preenchimento dessa lacuna, e tendo em vista que tal metodologia, embora muito pouco conhecida entre nós, tem sido bastante usada com sucesso em países como o Japão, este trabalho visa introduzir a discussão, dentro desse contexto, do método "Hoshin Kanri" ou, como traduzido por CAMPOS (1992), Gerenciamento pelas Diretrizes (GPD).

A discussão desse método se justifica pelo fato de que muitas empresas participantes do PBQP já começam a obter os resultados dos esforços com a implementação de modelos de Gestão da Qualidade, o que faz com que novas dificuldades se apresentem, pois os problemas a serem resolvidos se tornam mais complexos e envolvem atividades interfuncionais que permeiam toda a organização.

Essas empresas estão voltadas agora para a consolidação dos métodos de Gerenciamento da Qualidade, e as pessoas envolvidas necessitam de um direcionamento correto que as oriente no sentido adequado para a aplicação das técnicas por ele previstas. Caso contrário, a simples utilização das técnicas, sem um direcionamento adequado, pode levar esses métodos de Gerenciamento da Qualidade ao desprestígio e conseqüente esquecimento.

O "Hoshin Kanri” ataca justamente esse ponto, direcionando os esforços da organização de forma a apoiar cada indivíduo na aplicação das técnicas de melhoria da qualidade, evitando as possíveis dispersões que normalmente comprometem o sucesso da implementação de modelos de Gerenciamento da Qualidade.

Desta forma, a discussão que pretendemos iniciar busca esclarecer se o "Hoshin Kanri” realmente atende às necessidades da organização. Assim, apresentamos o método não como "a solução" para as organizações, mas como uma alternativa válida que deve ser analisada em relação a outras já experimentadas.

Para uma análise mais adequada do Gerenciamento pelas Diretrizes, o presente trabalho traça um paralelo com um modelo bastante tradicional, que foi desenvolvido com objetivos diferentes, mas que pode ser utilizado com a mesma finalidade, que é o Gerenciamento por Objetivos.

O Gerenciamento por Objetivos (GPO) é um método gerencial largamente utilizado por organizações em todo o mundo; principalmente em nosso contexto ocidental, em que é talvez a filosofia administrativa mais difundida nas últimas décadas. Por essa razão decidimos utilizá-la preferencialmente nesta análise, buscando tecer comparações que possam orientar o leitor na avaliação que se pretende realizar.

\section{Análise do momento atual}

$\mathrm{M}$ AIN (1994), em seu relatório sobre a evolução do Gerenciamento da Qualidade, apresenta importantes considerações e resultados obtidos em diversos casos de implementação do Gerenciamento da Qualidade. Entre as dificuldades encontradas, o relatório destaca que em geral os diretores-executivos das organizações ainda não entenderam que têm de assumir a 
liderança do processo de implementação. Essa argumentação é confirmada por TOLOVI (1994) em um estudo da realidade Brasileira.

BEISCHEL \& SMITH (1991) propõe que esse problema de liderança pode ser resolvido por meio de uma melhor ligação entre a alta administração e o chão-defábrica, destacando fatores críticos para o êxito como Qualidade do Produto, Atendimento ao Cliente, Custos, Flexibilidade e Moral, que devem ser comunicados de for- ma adequada ao pessoal do chão- de-fábrica. MINTZBERG (1994), com raciocínio semelhante ao já anteriormente apresentado por DONAVAN et al. (1978), destacam o mesmo problema, observando que o papel da liderança não está na definição de respostas, mas sim no estabelecimento da direção a ser seguida e na priorização dos problemas.

Estes trabalhos nos ajudaram na análise de como o GPD e o GPO se adaptam à realidade do ambiente de competição atual.

\section{O Gerenciamento por Objetivos}

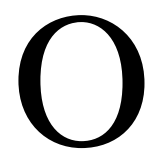

Gerenciamento por Objetivos é um método de gestão utilizado para integrar as funções planejamento e controle. KERR (1976) o define como sendo um processo de estabelecimento de objetivos de forma conjunta entre o gerente e seus subordinados.

O GPO busca um acordo formal entre gerente e subordinado englobando:
Os objetivos do subordinado para um dado período de tempo.

Os planos mediante os quais eles serão atingidos.

Normas para medir se os objetivos foram ou não atingidos.

Procedimentos para a reavaliação dos resultados.

A figura 1 representa esquematicamente o GPO:

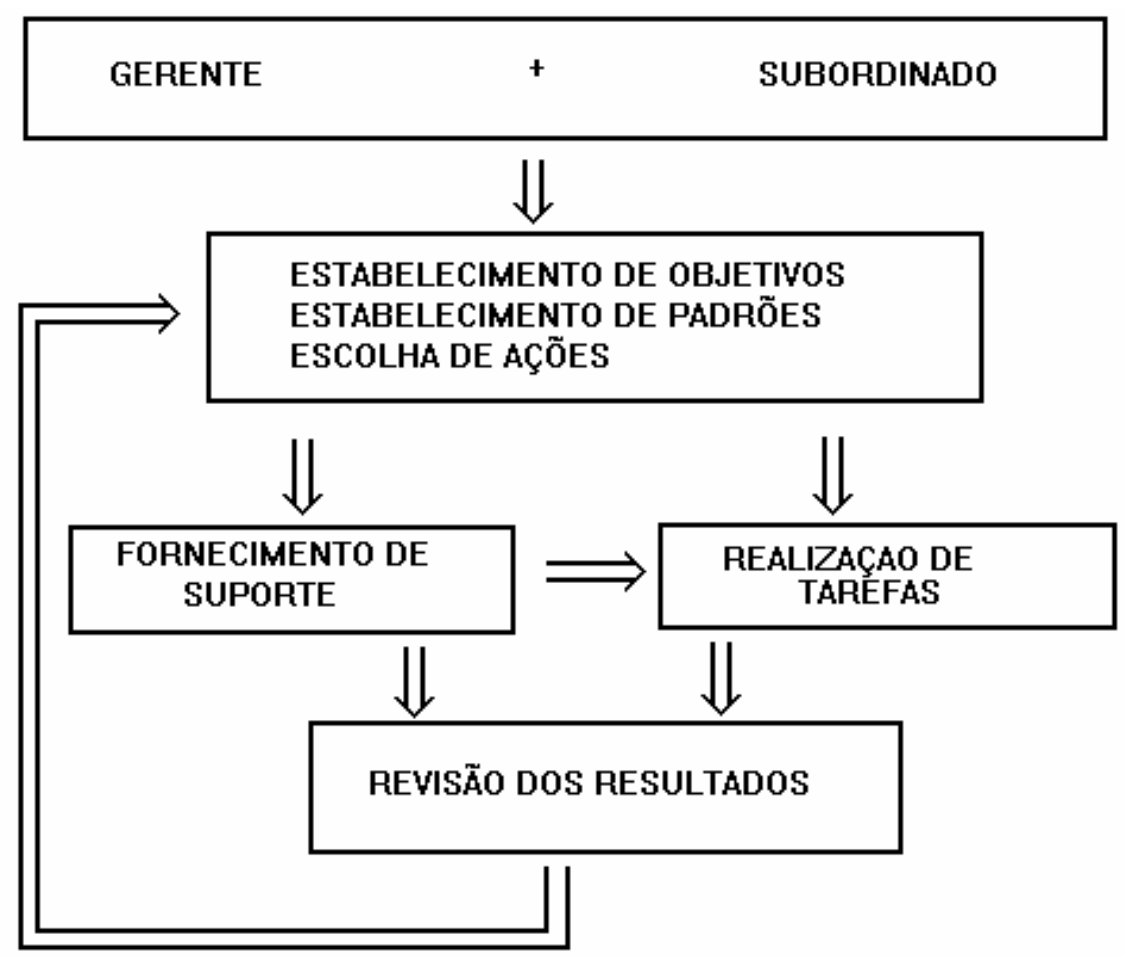

Figura 1: Estrutura para o Gerenciamento por Objetivos Adaptado de KERR (1976) 
É importante destacar a separação entre responsabilidades conjuntas e individuais.

$\mathrm{O}$ gerente e seu subordinado estabelecem o plano e controlam os resultados de forma conjunta. Esse envolvimento do subordinado habilita e encoraja o auto-controle. O resultado final do GPO é um documento formal que registra os objetivos do trabalho do subordinado, incluindo um cronograma e um conjunto de critérios de avaliação.

O GPO busca, portanto, a focalização dos esforços do subordinado para as tarefas críticas no atingimento dos objetivos e permite a comunicação face-a-face entre o gerente e seu subordinado, que tem a oportunidade de participar das decisões que afetam seu próprio trabalho.

\section{O "Hoshin Kanri" - Gerenciamento pelas Diretrizes}

A

KAO (1991) define o "Hoshin Kanri” como sendo uma abordagem sistemática para o gerenciamento da mudança em um processo crítico da organização.

CAMPOS (1992) afirma que o termo "Hoshin Kanri" foi traduzido erroneamente para o inglês como "Policy Deployment". Segundo este autor, o Gerenciamento pelas Diretrizes é um sistema administrativo, praticado por todas as pessoas da organização, que visa garantir a sobrevivência da organização à competição por meio de:
Estabelecimento da visão estratégica da organização com base na análise do sistema organização-ambiente e nas crenças e valores da organização.

Direcionamento da prática das atividades de controle da qualidade de todas as pessoas da organização segundo a visão estratégica estabelecida.

Este modelo de estabelecimento e disseminação da visão-estratégica por toda organização pode ser sintetizado esquematicamente na figura 2.

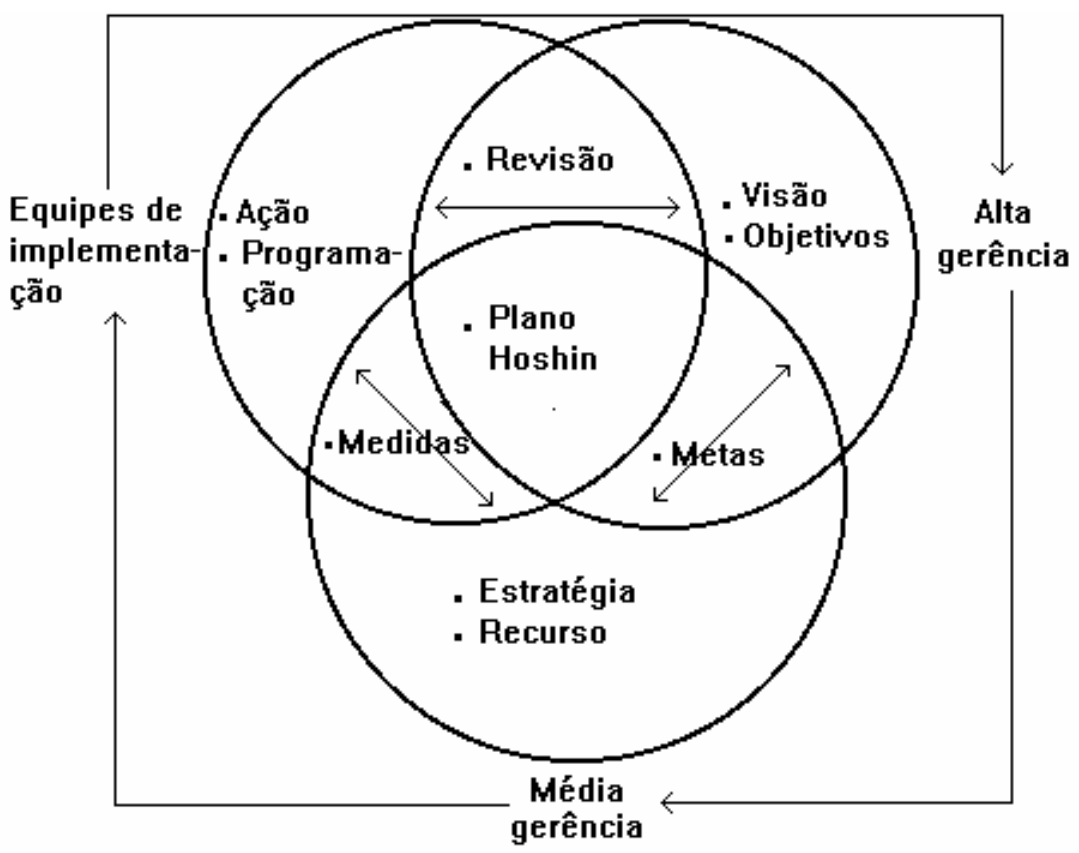

Figura 2: Fases do Gerenciamento pelas Diretrizes Adaptado de AKAO (1991)

Conforme apresentado, o processo se inicia com o estabelecimento, pela alta 
administração, da visão e dos objetivos fundamentais. A seguir, a média gerência define as metas, as estratégias e os recursos para o atingimento dessas metas. A última etapa do processo consta da montagem das equipes de implementação e da programação das atividades.

Estas três etapas apresentam pontos em comum, a destacar:

O estabelecimento das metas

A definição de medidas de desempenho

A revisão da visão estratégica

Nestes pontos é praticado o que AKAO (1991) designa como Catchball, que nada mais é que uma analogia com o jogo de baseball, em que os jogadores têm que ter muita precisão nos passes o que torna fundamental o treinamento da troca de passes. O "Hoshin Kanri” procura, com essa analogia, destacar que é fundamental a interação e a discussão para a decisão nesses pontos fundamentais do sistema. O que, de resto, não deveria ser novidade para ninguém

O GPD tem no seu desenvolvimento as seguintes fases:

\section{I)-Estabelecimento da Política da Qualidade:}

Nesta fase busca-se estabelecer a Política da Qualidade, que é o conjunto de diretrizes.

Estas são estabelecidas com quatro parâmetros:

Visão da equipe gerencial
Análise da situação competitiva

Análise do ambiente tecnológico

Oportunidades de mercado.

\section{II)-Definição de uma “estratégia” de manufatura:}

A estratégia de manufatura é o conjunto de ações que vão ser estabelecidas para o atingimento da Política de Qualidade. Esta não pode criar falsas expectativas, devendo estar baseada em métodos que sejam adequados à realidade da empresa.

\section{III)- Coleta e análise de fatos e dados:}

Esta fase trata da coleta de dados e da análise visando a seleção adequada de prioridades na aplicação dos métodos estabelecidos. O objetivo aqui é detectar pontos em que a aplicação correta dos métodos acelera o atingimento da Política da Qualidade, buscando orientar os esforços de cada um.

\section{IV)- Planejamento dos alvos e meios:}

Com base nas três fases anteriores, são estabelecidos nesta fase os parâmetros que permitirão a avaliação do atingimento da Política da Qualidade estabelecida. A definção dos alvos a serem atingidos e dos meios a serem utilizados deve dar prioridade às observações feitas nas fases anteriores, e resulta da contínua negociação entre os níveis de gerenciamento da empresa.

\section{Uma Análise Comparativa}

$\mathrm{F}$

Tazemos agora uma análise comparativa entre o Gerenciamento pelas Diretrizes e o Gerenciamento por Objetivos, a fim de proporcionar ao leitor uma visão das principais diferenças entre as duas abordagens.

Esta discussão se justifica, entre outras razões, pelo fato de que muitos possivelmente tendam a concluir que os métodos são muito parecidos, quando na verdade divergem em pontos fundamentais.

De fato, enquanto o GPO se preocupa basicamente em avaliar resultados, mormen- te financeiros, o GPD trata dos resultados e dos meios para atingí-los, considerando como ponto primordial as correlações entre os resultados. O GPO procura alcançar os resultados por intermédio do gerenciamento das pessoas envolvidas no processo, ao passo que o GPD focaliza os fatores critícos para o êxito, entre as quais se destaca o atingimento da satisfação dos clientes.

Ademais, enquanto o GPO é implementado de "cima para baixo", o GPD permite o fluxo de implementação nos dois sentidos, por meio do mecanismo denominado, por 
analogia catchball, que representa a interação entre os elementos do processo e que pode ser melhor entendido em BOWMAN (1995).

O GPO não integra as várias áreas funcionais, enquanto o GPD trata os problemas interfuncionais e estabeleca prioridades para as ações a serem implementadas.

Acreditamos que aí resida a principal vantagem do GPD, pois a ênfase no processo premite que os gerentes possam mudá-lo até se que produzam os resultados desejados, enquanto que a ênfase em tornar as pessoas responsáveis pelos resultados tende a fazer com que o processo não seja adequadamente estudado e entendido.

Na Tabela 1 são apresentados outros pontos de diferenciação entre as duas abordagens.

Tabela 1: Comparação entre Gerenciamento por objetivos e Gerenciamento pelas Diretrizes. Adaptado de SHIBA et al. (1993)

\begin{tabular}{|c|c|}
\hline $\begin{array}{l}\text { GERENCIAMENTO } \\
\text { POR OBJETIVOS }\end{array}$ & $\begin{array}{l}\text { GERENCIAMENTO } \\
\text { PELAS DIRETRIZES }\end{array}$ \\
\hline Ciclo de planejamento & Ciclo de planejamento \\
\hline 12 meses & 6 meses \\
\hline $\begin{array}{l}\text { Desdobra uma parte da meta de alto } \\
\text { nível para cada segmento, em cada nível } \\
\text { inferior. }\end{array}$ & $\begin{array}{l}\text { Desdobra as metas com diferentes me- } \\
\text { didas para cada segmento e em cada nível. }\end{array}$ \\
\hline $\begin{array}{l}\text { A média gerência é responsável pelo } \\
\text { fornecimento dos meios. }\end{array}$ & $\begin{array}{l}\text { A alta gerência sugere meios plausíveis } \\
\text { para as metas chave. }\end{array}$ \\
\hline Alguma negociação de metas & $\begin{array}{l}\text { Negociação de metas e meios baseados } \\
\text { em fatos e análises. }\end{array}$ \\
\hline Pouca monitoração dos meios. & $\begin{array}{l}\text { Algumas metas e medidas são voltadas } \\
\text { para o controle da aderência aos meios. } \\
\text { Análise das causas das falhas dos mei- } \\
\text { os do último ciclo de planejamento é usada } \\
\text { para melhorar os métodos propostos no novo } \\
\text { ciclo de planejamento }\end{array}$ \\
\hline $\begin{array}{l}\text { Recriminações do presidente com re- } \\
\text { lação a metas não atingidas e algumas } \\
\text { vezes substituição do gerente responsável. }\end{array}$ & $\begin{array}{l}\text { Diagnóstico presidencial com utiliza- } \\
\text { ção do ciclo P.D.C.A e sugestões de como } \\
\text { melhorar na próxima vez. }\end{array}$ \\
\hline $\begin{array}{l}\text { O novo gerente “deprecia” o sistema } \\
\text { anterior devido aos problemas do passado e } \\
\text { começa a planejar um novo sistema. }\end{array}$ & $\begin{array}{l}\text { O antigo gerente aprende com o passa- } \\
\text { do a fazer um melhor trabalho na próxima } \\
\text { vez. }\end{array}$ \\
\hline $\begin{array}{l}\text { Dependência de atividades não do- } \\
\text { cumentadas que os gerentes admitem ser } \\
\text { capazes de realizar. }\end{array}$ & $\begin{array}{l}\text { Atenção na documentação das ferra- } \\
\text { mentas necessárias e em institucionalizá-las } \\
\text { na companhia. }\end{array}$ \\
\hline
\end{tabular}


$\mathrm{O}$ presente trabalho está longe de pretender esgotar este assunto. Ao contrário, conforme já foi dito, busca apenas iniciar uma discussão a respeito.

A propósito, uma boa pergunta que poderia ser formulada, conveniência de se adotar o GPD, uma vez que ele tem similaridades com o GPO e este é bem mais conhecido e tradicional em nosso meio, e possivelmente até mais compatível com a nossa cultura. A resposta a esta questão estaria, a nosso ver, no fato de que o GPD reforça pontos pouco destacados pelo GPO, e que podem ser importantes dentro de um novo contexto empresarial, destacando-se :

aumento da interação entre a estratégia e o processo de implementação detalhamento do processo de implementação

busca do consenso, com a participação dos envolvidos e o uso de fatos e dados criação de uma estrutura formal de indicadores para 0 acompanhamento sistemático dos fatores que são críticos para a obtenção dos resultados desejados. Reconhecemos, por outro lado, que o esforço gerencial para implementação do GPD é substancialmente maior. Entretanto, nesta discussão inicial, julgamos mais importante avaliar a adequação e a viabilidade do uso deste modelo na realidade brasileira, para depois analisar as dificuldades ligadas à sua adaptação ao nosso contexto.

\section{Referências Bibliográficas:}

AKAO, Y.: Hoshin Kanri. Productivity Press, Cambridge, 1991.

BEISCHEL, M. \& SMITH, R.: "Linking the Shop Floor to the Top Floor". Management Accounting, October 1991

BOWMAN, J.: "Get Employees in the Game with Catchball”. Quality Progress, April 1995.

CAMPOS, V. F.: TQC Controle da Qualidade Total. Bloch Editores, Rio de Janeiro, 1992.

DONAVAN, N. B.; PAUL, R.; TAYLOR, J. W.: "The Reality Gap in Strategic Planning”. Harvard Business Review, May-June 1978

KERR, S.: "Overcoming the Dysfunctions of Management by Objetives". Management by Objetives, v.5, $\mathrm{n}^{\circ} 1,1976$
MAIN, J.: Guerras pela Qualidade: os sucessos e fracassos da revolução da qualidade. Editora Campus, 1994

MINTZBERG, H.: "The Fall and Rise of Strategic Planning“. Harvard Business Review, January-February, 1994

SHIBA, S. G. A. \& DAVID, W.: New American TQM. Productivity Press, Cambridge, 1993.

TOLOVI, J.: "Por que os programas de qualidade falham?”. Revista de Administração de Empresas, v34, número 6, 1994. 
POLICY DEPLOYMENT AND MANAGEMENT BY OBJECTIVES: A COMPARATIVE ANALYSIS

\section{Abstract}

Brazilian reality and the velocity of the diffusion process of the Total Quality Management model and its techniques have given rise to some complexities. Among them is the difficulty that top management has in making the Total Quality Managers actually achieve the desired objectives due to this implementation. This paper aims to open the discussion of the Policy Deployment method as an alternative to deal with these problems, in opposition to the more traditional and generally well known Management by Objectives.

Key-words: Total Quality Management, Policy Deployment, Management By Objectives. 